RESEARCH ARTICLE

\title{
Bioinformatics Analysis of Altered IncRNAs in Peripheral Blood Molecular Cells from Major Depressive Disorder (MDD) Patients
}

\author{
Guangyao $\mathrm{Li}^{1}$, Mingjun $\mathrm{He}^{2}$, Xiaoli $\mathrm{Zhu}^{2}$, Wei Niư ${ }^{3}$, Lingming kong ${ }^{2}$, Gaofeng $\mathrm{Yao}^{4}$, Shengdong Chen ${ }^{5}$, \\ Liyi Zhang ${ }^{2 *}$
}

1Psychological Counseling Center, No.904 Hospital of Chinese People's Liberation Army, Changzhou, People's Republic of China

2Prevention and Treatment Center for Psychological Diseases, No.904 Hospital of Chinese People's Liberation Army, Changzhou, People's Republic of China

3Department of Rehabilitation, No.904 Hospital of Chinese People's Liberation Army, Changzhou, People's Republic of China

4Psychiatry Department, Fuyang First People's Hospital, Anhui, People's Republic of China

5Department of Neurology, No.904 Hospital of Chinese People's Liberation Army, Changzhou, People's Republic of China

*Corresponding author: Liyi Zhang, Prevention and Treatment Center for Psychological Diseases, No.102 Hospital of Chinese People's Liberation Army, North Peace Road 55, People's Republic of China, Tel: +86 519 83064556; Fax: +86 519 83064560,E-mail: zly102@126.com

\section{Abstract}

Objectives: Based on the prior studies, altered IncRNAs in peripheral blood Molecular Cells (PBMC) from depression patients were chosen to perform informatics analysis for IncRNA target gene prediction and functional annotation.

Methods: Microarray was first used to screen dys regulated IncRNAs in the PBMCs of MDD patients, of which 10 IncRNAs were selected for quantitative real-time Reverse Transcription Polymerase Chain Reaction (RT-PCR) study, as well as bioinformatics analysis.

Results: According to microarray results, the top 10 IncRNAs with highest expression changes were chosen for further validation with qRT-PCR, 9 IncRNAs demonstrate significant down-regulation in expression levels (TCONS_L2_00001212, NONHSAT102891, TCONS_00019174, ENST00000566208, NONHSAG045500, ENST00000591189, ENST00000517573, NONHSAT034045, NONHSAT142707) (P < 0.05). IncRNA target gene prediction and functional annotation analysis showed a significant enrichments in several gene ontology (GO) biological process and Kyoto encyclopedia of genes and genomes (KEGG) pathways associated with nervous system and brain functions, suggesting that the differentially expressed IncRNAs may be involved in mechanism of MDD. Cytoscape network chart indicated that TCONS_L2_00001212, NONHSAT102891, TCONS_00019174, ENST00000566208,
\end{abstract}

NONHSAG045500, ENST00000591189, ENST00000517573, NONHSAT034045 and NONHSAT142707 also provide clues for the association of IncRNAs with MDD.

Conclusions: Altered expression of IncRNAs (TCONS L2_00001212, NONHSAT102891, TCONS_00019174, ENST00000566208, NONHSAG045500, ENST00000591189, ENST00000517573, NONHSAT034045 and NONHSAT142707) might be involved in MDD pathogenesis and may serve as noninvasive biomarker for MDD diagnosis.

\section{Keywords}

IncRNA, Depression, Target gene, Bioinformatics

\section{Introduction}

Major Depression Disorder, a mood disorder with high suicide risk [1], affects approximately 300 million people worldwide [2]. It has been suggested MDD was co-effected by genetics, personalities, cognitive, poor life event, biochemical disease, somatopathy and so on [3-5], of which, nearly $50 \%$ of the risk for depression is contributed to genetic factors [6-8].

Long non-coding RNA (IncRNA) was a molecular

Citation: He M, Zhu X, Niu W, Kong L, Yao G, et al. (2018) Bioinformatics Analysis of Altered IncRNAs in Peripheral Blood Molecular Cells from Major Depressive Disorder (MDD) Patients. Int J Blood Res Disord 5:034. doi.org/10.23937/2469-5696/1410034

Accepted: December 10, 2018: Published: December 12, 2018

Copyright: (C) $2018 \mathrm{He} \mathrm{M}$, et al. This is an open-access article distributed under the terms of the Creative Commons Attribution License, which permits unrestricted use, distribution, and reproduction in any medium, provided the original author and source are credited. 
with a length of over $200 \mathrm{nt}$, widely existing in animals, plants, yeasts, prokaryotes and viruses. A numerous study recently indicated IncRNA's function in genetics due to its conservative secondary structures and shear forms. A numerous study recently indicated IncRNA's function in genetics due to its conservative secondary structures and shear forms [9].

Because of the inconsistent between sequence and function of IncRNA, it is very hard to confirm their function. But fortunately, a large number of studies have indicated the association of IncRNA with mental disease [1014]. In this study, through long non-coding RNA (IncRNA) expression profiling and reverse transcription-polymerase chain reaction, 2115 down-regulated and 534 up-regulated IncRNAs were screened. 10 IncRNAs (TCONS L2_00001212, NONHSAT102891, TCONS_00019174, ENST00000566208, ENST00000414201, NONHSAG045500, ENST00000591189, ENST00000517573, NONHSAT034045 and NONHSAT142707) showed a marked differences $(P<0.01$ or $P<0.05)$.

Based on the prior studies, altered IncRNAs in peripheral blood Molecular Cells (PBMC) from Depression patients were chosen to perform informatics analysis for IncRNA target gene prediction and functional annotation.

\section{Materials and Methods}

\section{Subjects}

138 MDD patients who met the criteria of the Diagnostic and Statistical Manual of Mental Disorders, 5th Edition (DSM-V) were enrolled from No.102 Hospital of the Chinese People's Liberation Army from May 2014 to February 2015. Diagnoses were made independently by three attending psychiatric doctors using the Chinese version of the modified Structured Clinical Interview for DSM-V. All patients were presenting for their first visit without any prior clinical treatment. They had not taken antipsychotic, antidepressant, anxiolytic drugs for at least three months before study enrollment, had no previous history of organic disease (such as heart disease, diabetes, and Parkinson's disease) and no other psychiatric disorders.

The 63 healthy controls were recruited from No.102 Hospital of the Chinese People's Liberation Army from May 2014 to February 2015, without any family history of major psychiatric disorders (including schizophrenia, bipolar disorder and anxiety disorders) and without any history of severe traumatic events in the past month.

Prior to study entry, all risks, benefits and potential adverse events associated with participation in the study were explained to the participants and their legal guardians, who provided written informed consent according to a protocol approved by the Ethical Committee for Medicine of No.102 Hospital of Chinese People's Liberation Army, PR, China.

\section{Experimental methods}

RNA extraction: Whole blood $(5 \mathrm{ml})$ was collected in EDTA anticoagulant tube from each subject and processed within 1 hour. PBMCs were isolated from the blood through density gradient centrifugation and stored at $-80{ }^{\circ} \mathrm{C}$ until use. Total RNAs were extracted from the PBMCs with Trizol (Invitrogen, Carlsbad, CA, USA) and the RNeasy kit (Qiagen, Hilden, Germany) according to the manufacturer's protocol, quantified by Nano Drop ND-2000 (Thermo Scientific, Delaware, ME, USA), DNase treated (Turbo DNase, Life Technologies) and reverse transcribed (Superscript III; Invitrogen). The integration of RNA was assessed using Agilent Bioanalyzer 2100 (Agilent Technologies).

LncRNA microarray expression profiling: RNA samples were used for IncRNA microarray profiling. LncRNA expression was measured by Human IncRNA 3.0 array (Arraystar, Santa Clara, CA, USA). The sample labeling, microarray hybridization and washing were performed based on the manufacturer's standard protocols. Afterwards, the labeled RNAs were hybridized onto the microarray. Having washed and stained the slides, the arrays were scanned by the Agilent Scanner G2505C (Agilent). The scanned images were analyzed using Feature Extraction software (version 10.7.1.1, Agilent Technologies) and Genespring software (version 12.5; Agilent Technologies).

Real-time quantitative reverse-transcription PCR (qRT-PCR): According to microarray results, the top 10 IncRNAs with the highest differentially expression were chosen for further validation with qRT-PCR. Blood samples from 69 GAD patients and 41 controls were used to validate the candidate IncRNAs. Total RNAs were isolated from the PBMCs using Trizol reagent (Invitrogen ${ }^{\circledR}$, USA) for quantitative detection of IncRNA. Complementary RNA was synthesized using the Reverse Transcription TaqMan RNA Reverse Transcription Kit (Applied Biosystems, inc., USA) according to the manufacturer's instructions. Real-time PCR was performed using Applied Biosystems 7900 HT RealTime PCR System (Applied Biosystems, Inc., USA). Data were collected using the SDS 2.3 software (Applied Biosystems, Inc.) and Data Assist v3.0 software. After normalized to $\beta$-Actin, the expression levels of IncRNAs were calculated using the $2-\Delta \Delta \mathrm{Ct}$ method.

Data acquisition: Feature Extraction (version 10.7.1.1, Agilent Technologies) was applied to act on original image for extracting original data, then Genespring.

\section{Results}

Qrt-PCR result showed that TCONS_L2_00001212, NONHSAT102891, TCONS_00019174, ENST00000566208, NONHSAG045500, ENST00000591189, ENST00000517573, NONHSAT034045 and NONHSAT142707 were identified as significantly different from the control group $(\mathrm{P}<0.01$ 
Table 1: Comparison of IncRNA expression levels between MDD group and control group ( $\Delta \mathrm{Ct}, \bar{X} \pm \mathrm{s}$ ).

\begin{tabular}{|l|l|l|l|l|l|}
\hline IncRNA & GAD $(\mathbf{n}=\mathbf{1 3 8})$ & Control group $\mathbf{( n = 6 3 )}$ & Fold change & Up-down & $\boldsymbol{P}$-value \\
\hline TCONS_L2_00001212 & $6.91 \pm 2.11$ & $5.78 \pm 2.36$ & 5.686522 & Down & 0.0067 \\
\hline NONHSAT102891 & $7.12 \pm 2.21$ & $6.15 \pm 2.41$ & 3.558301 & Down & 0.0242 \\
\hline TCONS_00019174 & $6.95 \pm 2.46$ & $5.77 \pm 2.78$ & 3.775364 & Down & 0.0153 \\
\hline ENST00000566208 & $6.36 \pm 2.51$ & $5.34 \pm 2.70$ & 3.697919 & Down & 0.0373 \\
\hline ENST00000414201 & $6.22 \pm 2.60$ & $5.26 \pm 2.67$ & 5.115068 & Down & 0.0559 \\
\hline NONHSAG045500 & $8.24 \pm 2.52$ & $7.23 \pm 2.57$ & 3.514152 & Down & 0.0376 \\
\hline ENST00000591189 & $4.84 \pm 1.51$ & $4.15 \pm 1.81$ & 4.610542 & Down & 0.0243 \\
\hline ENST00000517573 & $7.50 \pm 2.69$ & $6.34 \pm 2.69$ & 3.242618 & Down & 0.0237 \\
\hline NONHSAT034045 & $7.01 \pm 2.71$ & $5.98 \pm 2.76$ & 3.405084 & Down & 0.0457 \\
\hline NONHSAT142707 & $9.14 \pm 2.57$ & $7.97 \pm 2.76$ & 5.130027 & Down & 0.0193 \\
\hline
\end{tabular}

Table 2: Enrichment items of IncRNA target genes in GO.

\begin{tabular}{|l|l|l|l|}
\hline GO number & Item & $\boldsymbol{P}$-value & Gene name \\
\hline GO: $\mathbf{0 0 1 0 4 6 7}$ & gene expression & $3.76 \mathrm{e}-05$ & splicing factor 3a, subunit 1, 120 kDa \\
\hline GO: $\mathbf{0 0 0 7 5 9 6}$ & blood coagulation & $7.1 \mathrm{e}-04$ & CAP, adenylate cyclase-associated protein 1 (yeast) \\
\hline GO: $\mathbf{0 0 4 4 2 6 7}$ & cellular protein metabolic process & $3.35 \mathrm{e}-06$ & defender against cell death 1 \\
\hline GO: $\mathbf{0 0 1 6 0 3 2}$ & viral reproduction & $9.45 \mathrm{e}-04$ & nucleoporin $50 \mathrm{kDa}$ \\
\hline GO: $\mathbf{0 0 3 0 1 6 8}$ & platelet activation & $3.21 \mathrm{e}-05$ & CAP, adenylate cyclase-associated protein 1 (yeast) \\
\hline GO: $\mathbf{0 0 0 6 4 1 2}$ & translation & $1.46 \mathrm{e}-04$ & ribosomal protein L 10-like \\
\hline GO: $\mathbf{0 0 4 4 4 1 9}$ & interspecies interaction between organisms & $9.53 \mathrm{e}-03$ & acyl-CoA thioesterase 8 \\
\hline GO: $\mathbf{0 0 0 7 4 1 1}$ & axon guidance & $7.55 \mathrm{e}-03$ & myosin, light chain 12B, regulatory \\
\hline GO: $\mathbf{0 0 3 1 0 1 8}$ & endocrine pancreas development & $3.18 \mathrm{e}-09$ & glucokinase (hexokinase 4) \\
\hline GO: $\mathbf{0 0 0 6 4 1 4}$ & translational elongation & $1.29 \mathrm{e}-09$ & eukaryotic translation elongation factor 1 alpha 1 \\
\hline GO: $\mathbf{0 0 1 9 0 5 8}$ & viral infectious cycle & $8.39 \mathrm{e}-10$ & ribosomal protein L 3-like \\
\hline GO: $\mathbf{0 0 0 6 4 1 5}$ & translational termination & $1.83 \mathrm{e}-09$ & ribosomal protein L 3-like \\
\hline GO: $\mathbf{0 0 1 9 0 8 3}$ & viral transcription & $7.42 \mathrm{e}-10$ & ribosomal protein L 3-like \\
\hline GO: $\mathbf{0 0 4 8 0 1 1}$ & nerve growth factor receptor signaling pathway & $2.29 \mathrm{e}-02$ & BCL2-like 11 (apoptosis facilitator) \\
\hline
\end{tabular}

Table 3: Enrichment items of IncRNA target genes in KEGG.

\begin{tabular}{|l|l|l|l|}
\hline GO number & Item & $\boldsymbol{P}$-value & Gene name \\
\hline $\mathbf{0 3 0 1 0}$ & ribosome & $2.24 \mathrm{e}-09$ & ribosomal protein L 10-like \\
\hline $\mathbf{0 4 6 7 0}$ & leukocyte transendothelial migration & $4.91 \mathrm{e}-03$ & myosin, light chain 12B, regulatory \\
\hline $\mathbf{0 5 4 1 4}$ & dilated cardiomyopathy & $1.29 \mathrm{e}-02$ & Calcium channel, voltage-dependent, gamma subunit 3 \\
\hline $\mathbf{0 4 1 4 6}$ & peroxisome & $1.43 \mathrm{e}-02$ & acyl-CoA thioesterase 8 \\
\hline $\mathbf{0 4 9 7 0}$ & salivary secretion & $3.11 \mathrm{e}-02$ & Adenylate cyclase 9 \\
\hline $\mathbf{0 4 6 1 2}$ & antigen processing and presentation & $1.43 \mathrm{e}-02$ & cAMP responsive element binding protein 1 \\
\hline $\mathbf{0 5 1 1 0}$ & & $1.63 \mathrm{e}-02$ & Sec61 beta subunit \\
\hline $\mathbf{0 5 3 4 0}$ & primary immunodeficiency & $2.75 \mathrm{e}-02$ & Interleukin 2 receptor, gamma \\
\hline
\end{tabular}

or $\mathrm{P}<0.05$, Table 1). Qrt-PCR result showed that TCONS L2_00001212, NONHSAT102891, TCONS_00019174, ENST00000566208, NONHSAG045500, ENST00000591189, ENST00000517573, NONHSAT034045 and NONHSAT142707 were identified as significantly different from the control group $(P<0.01$ or $P<0.05$, Table 1$)$.

IncRNA target gene prediction and functional annotation analysis showed a significant enrichments in several gene ontology (GO) associated with nervous system and brain functions, suggesting that the differentially expressed IncRNAs may be involved in mechanism of MDD (Table 2).

IncRNA target gene prediction and functional annotation analysis showed a significant enrichments in Kyoto encyclopedia of genes and genomes (KEGG) pathways associated with nervous system and brain functions, suggesting that the differentially expressed IncRNAs may be involved in mechanism of MDD. Cytoscape network chart indicated that TCONS_L2_00001212, NONHSAT102891, TCONS_00019174, ENST00000566208, NONHSAG045500, ENST00000591189, ENST00000517573, NONHSAT034045 and NONHSAT142707 also provide clues for the association of IncRNAs with MDD (Table 3).

\section{Discussion}

Given the evidence that estimated $90 \%$ of noncoding proteins in human genome have important role in genetics, not just "translational noise", it seems reasonable that IncRNA may play an important role in regulation of transcription and post-transcription [15]. Research also demonstrated that IncRNA acts as the molecular scaffold, making sense for the transmit of biological signals, interaction of molecules, and the specificities and dynamics of signal regulation [16]. Until now, MDD is a mental illness with the functional changes of neurotransmitter, and more and more evidences have revealed the association of differentially expressed 
IncRNAs with neurodevelopment, neurodegeneration and neuroimmunological disorders [17-19].

Given the consistent evidences, it makes sense to further explore the biological function on the nine down-regulated IncRNAs.

LncRNA is found to serve as a very important epigenetic regulation factor in human genome, regulating DNA methylation, histone modification and chromatin remodeling through epigenetic, transcriptional regulation and post-transcription regulation, making the gene silencing or activation. In this study, at first, we re-tested the nine up-regulated differentially expressed IncRNAs through gene ontology (GO), indicated that the IncRNAs expression in MDD had a enrichment in translation, DNA dependency transcriptional regulation, axon elongation, dendritic morphology, cell differentiation, long-term memory, RNA transfer, neurons development, and so on. This was similar to the existing results. A study demonstrated that MDD was significantly enriched in central system, including axon elongation, dendritic morphology, cell differentiation, long-term memory [20].

Simultaneously, we also perform Kyoto encyclopedia of genes and genomes (KEGG) pathways, and the result illustrated that there is a enrichment in central nervous system. Among which, the low-regulation of Wnt signaling pathway may cause hippocampal neuron denaturation [21], $\mathrm{Ca}^{2+} /$ calmodulin-dependent protein kinase IV and MAPK signaling pathway are closely associated with the regulation processes of depression behavior [22-25]; biological analysis related to molecular pathway of depression revealed the enrichment in VEGF signaling pathway, GnRH signaling pathway and LTP pathway [26]. In addition to this, TCONS_L2_00001212, NONHSAT102891, TCONS_00019174, ENST00000566208, NONHSAG045500, ENST00000591189, ENST00000517573, NONHSAT034045 and NONHSAT142707 were seen to be posited in the centre of network, thus it is considered that these nine IncRNAs could be biomarkers of MDD.

In conclusion, we speculate that TCONS_L2_00001212, NONHSAT102891, TCONS_00019174, ENST00000566208, NONHSAG045500, ENST00000591189,ENST00000517573, NONHSAT034045 and NONHSAT142707 could be potential indicators for the diagnosis of MDD. The clinical significance of this study was given advice for the early forecast and diagnosis of MDD.

\section{References}

1. Subramaniam M, Abdin E, Seow EL, Picco L, Vaingankar JA, et al. (2014) Suicidal ideation, suicidal plan and suicidal attempts among those with major depressive disorder. Ann Acad Med Singapore 43: 412-421.

2. Fleischmann A, De Leo D (2014) The World Health Organization's report on suicide: A fundamental step in worldwide suicide prevention. Crisis 35: 289-291.

3. Avenevoli S, Swendsen J, He JP, Burstein M, Merikangas KR (2015) Major depression in the national comorbidity survey-adolescent supplement: Prevalence, correlates, and treatment. J Am Acad Child Adolesc Psychiatry 54: 37-44.

4. M Serra-Blasco, $X$ Goldberg, EM Aguilar, Muriel Vicent, Guillem Navarra, et al. (2017) Effects of early and adult stress on symptom severity and neurocognitive function of major depression. Biological Psychiatry 81: 76-77.

5. X Huang, YL Luo, YS Mao, JL Ji (2016) The link between long noncoding RNAs and depression. Prog Neuropsychopharmacol Biol Psychiatry.

6. Cui X, Niu W, Kong L, He M, Jiang K, et al. (2017) Long noncoding RNA expression in peripheral blood mononuclear cells and suicide risk in Chinese patients with major depressive disorder. Brain Behav 7: e00711.

7. Cui X, Niu W, Kong L, He M, Jiang K, et al. (2017) Can IncRNAs be indicators for the diagnosis of early onset or acute schizophrenia and distinguish major depressive disorder and generalized anxiety disorder?-A cross validation analysis. Am J Med Genet B Neuropsychiatr Genet 174: 335-341.

8. Cui X, Niu W, Kong L, He M, Jiang K, et al. (2017) Long noncoding RNAs: New evidence for overlapped pathogenesis between major depressive disorder and generalized anxiety disorder. Indian J Psychiatry 59: 83-87.

9. Clark BS, Blackshaw S (2014) Long non-coding RNAdependent transcriptional regulation in neuronal development and disease. Front Genet 5: 164.

10. Song HT, Sun XY, Zhang L, Zhao L, Guo ZM, et al. (2014) A preliminary analysis of association between the down-regulation of microRNA-181b expression and symptomatology improvement in schizophrenia patients before and after antipsychotic treatment. J Psychiatr Res 54: 134-140.

11. Fan HM, Sun $X Y$, Guo W, Zhong AF, Niu W, et al. (2014) Differential expression of microRNA in peripheral blood mononuclear cells as specific biomarker for major depressive disorder patients. J Psychiatr Res 59: 45-52.

12. Sheng-dong Chen, Xin-yang Sun, Wei Niu, Ling-ming Kong, Ming-jun He, et al. (2016) Correlation between the level of microRNA expression in peripheral blood mononuclear cells and symptomatology in patients with generalized anxiety disorder. Science Direct 69: 216-224.

13. Fan H, Niu W, He M, Kong L, Zhong A, et al. (2015) Bioinformatics analysis of differently expressed microRNAs in anxiety disorder. Med Genet 32: 641-646.

14. X Cui, X Sun, W Niu, L Kong, M He, et al. (2016) Long non-coding RNA: Potential diagnostic and therapeutic biomarker for major depressive disorder. Med Sci Monit 22: 5240-5248.

15. DH Mathews, DH Turner, M Zuker (2016) RNA secondary structure prediction. Bmc Bioinformatics 67.

16. J Li, M Zhang, G An, Q Ma (2016) LncRNA TUG1 acts as a tumor suppressor in human glioma by promoting cell apoptosis. Exp Biol Med 241: 644-649.

17. Qureshi IA, Mehler MF (2013) Long non-coding RNAs: Novel targets for nervous system disease diagnosis and therapy. Neurotherapeutics 10: 632-646.

18. Rao SQ, Hu HL, Ye N, Shen Y, Xu Q (2015) Genetic variants in long non-coding RNA MIAT contribute to risk of paranoid schizophrenia in a Chinese Han population. Schizophr Res 166: 125-130.

19. Liu Z, Li X, Sun N, Xu Y, Meng Y, et al. (2014) Microarray profiling and co-expression network analysis of circulating IncRNAs and mRNAs associated with major depressive disorder. PLoS One 9: e93388. 
20. Debra Walker, Jane Leach (2014) Anxiety: Etiology, treatment, and christian perspectives. J Christ Nurs 31: 84-91.

21. YH Zhao, TF Ji, Q Luo, JL Yu (2017) Long non-coding RNA H19 induces hippocampal neuronal apoptosis via Wnt signaling in a streptozotocin-induced rat model of diabetes mellitus. Oncotarget 8: 64827-64839.

22. WJ Su, Y Zhang, Y Chen, H Gong, YJ Lian, et al. (2017) NLRP3 gene knockout blocks NF-kB and MAPK signaling pathway in CUMS-induced depression mouse model. Behav Brain Res 322: 1-8.

23. Nadeem A, Ahmad SF, Al-Harbi NO, Fardan AS, ElSherbeeny AM, et al. (2017) IL-17A causes depressionlike symptoms via NFkB and p38MAPK signaling pathway in mice: Implications for psoriasis associated depression. Cytokine 97: 14-24.

24. G Zheng, M Pan, W Jin, G Jin, Y Huang (2017) MicroRNA$135 \mathrm{a}$ is up-regulated and aggravates myocardial depression in sepsis via regulating p38 MAPK/NF-kB pathway. Int Immunopharmacol 45: 6-12.

25. LB Bergantin, A Caricati-Neto (2017) Advances for the pharmacotherapy of depression- Presenting the rising star: $\mathrm{Ca} 2+/ \mathrm{cAMP}$ signaling interaction. Open access text.

26. Puighermanal E, Busquets-Garcia A, Gomis-González M, Marsicano G, Maldonado R, et al. (2013) Dissociation of the pharmacological effects of THC by mTOR Blockade. Neuropsychopharmacol 38: 1334-1343. 